\title{
Enhancement of Antibacterial Activity of Short Tryptophan-rich Antimicrobial Peptide Pac-525 by Replacing Trp with His(chx)
}

\author{
Mija Ahn, ${ }^{a}$ Ganesan Rajasekaran, ${ }^{\dagger, a}$ Gunasekaran P, ${ }^{\S}$ Eun kyoung Ryu, Ga-hyang Lee, \\ Jae-Kyung Hyun, ${ }^{\ddagger}$ Chaejoon Cheong, Nam-Hyung Kim, ${ }^{\S}$ Song Yub Shin, ${ }^{\dagger, *}$ and Jeong-Kyu Bang ${ }^{*}$
}

\author{
Division of Magnetic Resonance, Korea Basic Science Institute,Chungbuk 363-883, Korea. *E-mail: bangjk@kbsi.re.kr \\ †Department of Bio-Materials, Graduate School and Department of Cellular \& Molecular Medicine, School of Medicine, \\ Chosun University, Gwangju 501-759, Korea.*E-mail: syshin@chosun.ac.kr \\ ${ }^{\S}$ Molecular Embryology Laboratory, Department of Animal Sciences, Chungbuk National University, \\ Cheongiu, Chungbuk 361-763, Korea \\ ${ }^{*}$ College of Pharmacy, Chungbuk National University, Cheongju, Chungbuk 361-763, Korea \\ ${ }^{\star}$ Division of Electron Microscopic Research, Korea Basic Science Institute, Daejeon 305-333, Korea \\ Received May 15, 2014, Accepted May 30, 2014
}

\begin{abstract}
Trp residue is considered as one of the important constituents in antimicrobial peptides (AMPs) as it presence secured good activities in many cases. However, it is preferable to be changed because of their sensitivity towards light. We have synthesized the short Trp-rich AMP Pac-525 and its analogues to investigate the possibility of His(chx) as possible replacement analogue for Trp in AMPs. Based on the assay result of the antibacterial activity including anti-MRSA activity, His(chx) is considered as good candidate for the Trp replacement. Through these study, we found that His(chx) had several merits to design therapeutic antimicrobial agents compare to Trp in terms of i) increasing antibacterial activity without hemolytic activity, ii) successful in designing the short peptide (only four residues), iii) having anti-MRSA activity, iv) overcoming the light sensitivity. Furthermore, transmission electron microscopy (TEM) and dye leakage experiments suggested that P11 and P16 containing His(chx) kill bacteria via forming pore/ion channels on bacterial cell membranes.
\end{abstract}

Key Words : Antimicrobial peptides, MRSA activity, Pac- 525, Light sensitivity, Short Trp-rich peptide

\section{Introduction}

For past few decades antibiotics have been a major area of focus in pharmaceutical chemistry because of their biological importance as drug substances as proficient of fighting against infectious disease caused by microorganisms. ${ }^{1}$ Nowdays, antibiotics are among the most frequently suggested drugs used in human medicine. ${ }^{2}$ However, treatment of intracellular bacterial infection remains both a medical and economic challenge because microorganisms possessing the ability to thrive and maintain themselves in cells simply by taking transient shelter therein, are indeed shielded from cellular defense as well as many antibiotics. ${ }^{3}$ Hence the intracellular bacteria are very harmful for the host cells and also behaves as reservoir for reinfection and recurrence. Subsequently the poor act of antibiotics on intracellular bacteria, leads to the foster in resistant mutants. Prolong, excessive and irresponsible intake of antibiotics also results in the steep and steady raise of antibiotic-resistant microorganisms known as "nightmare bacteria" which is a serious and imminent threat to global health care. ${ }^{4}$ Hence, there is a vital need for design and discovery of fast-acting, effective newer therapeutic methods to effectively kill antibioticresistant microorganisms is highly imperative.

\footnotetext{
${ }^{\mathrm{a}}$ These authors contributed equally to this work.
}

In the recent years, antimicrobial peptides (AMPs) are recognized as new class of antibiotics, are proficient of delivering potent broad-spectrum of antimicrobial activity against pathogens at the physiological concentration, while most defensins fails to display the activity. ${ }^{5}$ AMPs are produced by innate immune system as a firm acting weapon fighting against invading pathogens of many creatures such as plants, insects, amphibians and mammals in response to the foreign bodies such as bacteria, viruses, or fungi. ${ }^{6}$ AMPs are considered as potential candidates as peptide drugs because their relative short in length, very fast, efficient activity against microbes, and low toxicity to mammals cells. ${ }^{7}$

Over a period of years, several hundred investigations are present related to synthesis and characterizations of AMPs. ${ }^{8}$ Among them, Trp-rich AMPs have been studied very intensively since they exhibited the potent activity even at short peptide length (less than 6 residues). ${ }^{9}$ The activity of this class of AMPs is recognized to the fact that enhanced peptide-membrane interactions created as result of Trp motif, has a discrete preference over the interfacial region of lipid bilayers and also exhibits the distinct cation- $\pi$ interactions..$^{10}$ It is obvious that the indole ring of Trp provides facile access for implicating itself in peptide and protein folding in aqueous solution because of the native and nonnative hydrophobic interactions. ${ }^{11}$ Despite these potential 
advantageous features of indole ring of Trp, still there are some challenges restricts their applications such as, (i) the association of cationic antibacterial peptides with cell membranes generally causes not only the lysis of bacteria but also hemolysis, (ii) indolyl aromatics are very sensitive to light which has pushed the limits of both the preparation and conservation of AMP as therapeutic agents.

It was well known that the high ratio of Trp content in AMPs were not an essential feature for exhibiting natural antibacterial activity but rather the size and shape of the aromatic moiety linked with the amino acid act as determining force to antibacterial activity. For instances, substitution of various amino acids irrespective of their nature for Trp residue in lactoferricin derivatives, revealed the remarkable progress in the antibacterial activity. ${ }^{12}$ Furthermore, it has been proven that the hydrophobicity determines the hemolytic activity of AMPs. For example, replacement of all five Trp residues in indolicidin with Phe results in reducing the hemolytic activity, although the antibacterial activity was retained as same. ${ }^{13}$ The above results induces that replacement of natural or unnatural aromatic amino acids which are relatively similar or larger volumes than Trp into AMPs can enhance their antibacterial activity meanwhile reduce their hemolytic activity.

It is pertinent to note that histidine (His) possessing imidazole ring is more susceptible to implicate itself in peptide as well as in protein folding in the similar fashion as behave as indole moiety in Trp. ${ }^{11}$ Furthermore, hydrophobicity of the ring can be achieved by introducing pendant alkyl tails at $N(\pi)-, N(\tau)$ positions. Consequently, the amphiphillic amino acid histidine can be considered as an essential alternate for the logical replacement of tryptophan to examine the influence of charge to bulk ratio modification.

Previously, we designed and synthesized dicyclohexylsubstituted His derivatives known as His(chx), in favour of increasing the hydrophobicity of AMPs. ${ }^{14}$ As part of our research program embarked on the design and the study of AMPs, the present work stems as to study the possibility of His(chx) as possible replacement analogue for Trp in Pac525 which is a Trp-rich peptide ${ }^{15}$ and the following studies were made namely, antibacterial activity and anti-methicillinresistant Staphylococcus aures (MRSA) activity. Moreover we also investigate whether there is a substantial effect on the secondary structures of the analogues on the basis of their CD spectra by the substitution of His(chx) into Trp position. Also, the molecular mechanism of antibacterial activity was examined by transmission electron microscopy (TEM) and dye leakage from bacterial membrane-mimicking lipid vesicles.

\section{Experimental}

Materials. Rink amide 4-methylbenzhydrylamine (MBHA) resin and 9-fluorenylmethoxycarbonyl (Fmoc) amino acids were obtained from Calbiochem-Novabiochem (La Jolla, CA). Other reagents used for peptide synthesis included trifluoroacetic acid (TFA: Sigma), piperidine (Merck), 1-O-
Benzotriazole- $N, N, N^{\prime}, N^{\prime}$-tetramethyl-uronium-hexafluorophosphate (HBTU: Aldrich), $N$-hydroxybenzotriazole hydrate (HOBt: Aldrich), N,N-diisopropylethylamine (DIEA: Aldrich) and dimethylformamide (DMF, peptide synthesis grade: Biolab). All other reagents were of analytical grade. The buffers were prepared in double glass-distilled water.

Synthesis and Characterization of Peptides. All peptides were prepared by Fmoc SPPS methods using Rink amide resin with an initial loading of $0.61 \mathrm{mmol} / \mathrm{g}$, unless otherwise noted. Resins were swollen in DMF for 45 min prior to synthesis. For sequence extension, the Fmoc-protected amino acid ( 5 eq.) was activated by treatment with HBTU (5.0 eq.), HOBt (5.0 eq.) and DIEA (10 eq.) in DMF (2 mL) for $2 \mathrm{~min}$. This solution was added to the free amine on resin, and the coupling reaction was allowed to proceed for $1 \mathrm{~h}$ with Vortex stirring. After washing with DMF, Fmoc deprotection was achieved with $20 \%$ piperidine in DMF $(1 \times 10 \mathrm{~min}, 2 \times 3$ $\min )$. The resin was washed once again, and the process was repeated for the next amino acid and finally the resin was washed with DMF, methanol, dichloromethane and ether, and then dried under vacuum. Linear peptides were cleaved from the resin with 5\% triisopropylsilane (TIS) and $5 \% \mathrm{H}_{2} \mathrm{O}$ in trifluoroacetic acid (TFA, approximately $2 \mathrm{~mL}$ of TFA per $100 \mathrm{mg}$ of resin) for $2 \mathrm{~h}$. The cleavage cocktail was mixed with cold ether to precipitate the peptide and then filtered. Preparative reverse-phase HPLC analysis (RP-HPLC) was carried out on the preparative Vydac $\mathrm{C}_{18}$ column $(15 \mu \mathrm{m}, 20$ $\mathrm{mm} \times 250 \mathrm{~mm}$ ) using $0-90 \%$ water/acetonitrile gradient in the presence of $0.05 \%$ TFA. The final purity of the peptides $(>95 \%)$ was assessed by RP-HPLC on an analytical Vydac $\mathrm{C}_{18}$ column $(4.6 \mathrm{~mm} \times 250 \mathrm{~mm}, 300 \AA$, $5 \mu \mathrm{m}$ particle size $)$. The molecular masses of purified peptides were determined using matrix-assisted laser-desorption ionization-time-of-flight mass spectrometry (MALDI-TOF MS) (KBSI, Ochang).

Antibacterial Activity (MIC). The antibacterial activity of the peptides against two Gram-positive bacterial strains and two Gram-negative bacterial strains was examined by using the broth microdilution method in sterile 96-well plates. Aliquots $(100 \mu \mathrm{L})$ of a bacterial suspension at $2 \times 10^{6}$ $\mathrm{CFU} / \mathrm{mL}$ in $1 \%$ peptone were added to $100 \mu \mathrm{L}$ of the peptide solution (serial 2-fold dilutions in $1 \%$ peptone). After incubation for $18-20 \mathrm{~h}$ at $37^{\circ} \mathrm{C}$, bacterial growth inhibition was determined by measuring the absorbance at $600 \mathrm{~nm}$ with a Microplate Autoreader EL 800 (Bio-Tek Instruments, VT). The minimal inhibitory concentration (MIC) was defined as the lowest peptide concentration that causes $100 \%$ inhibition of microbial growth. Two types of Gram-positive bacteria (Staphylococcus epidermidis [KCTC 1917] and Staphylococcus aureus [KCTC 1621]) and two types of Gram-negative bacteria (Escherichia coli [KCTC 1682] and Pseudomonas aeruginosa [KCTC 1637]) were procured from the Korean Collection for Type Cultures (KCTC) at the Korea Research Institute of Bioscience and Biotechnology (KRIBB). Methicillin-resistant Staphylococcus aureus (MRSA) (CCARM 3089, CCARM 3090 and CCARM 3095) were obtained from the Culture Collection of Antibiotic-Resistant Microbes (CCARM) at Seoul Women's University (Seoul, 
Korea).

Hemolytic Activity. Hemolytic activity of peptides was tested against human red blood cells (hRBCs). Fresh human red blood cells (hRBCs) were centrifuged, washed three times with PBS (35 mM phosphate buffer, $0.15 \mathrm{M} \mathrm{NaCl}, \mathrm{pH}$ 7.4), dispensed into 96-well plates as $100 \mathrm{~mL}$ of $4 \%(\mathrm{v} / \mathrm{v})$ $\mathrm{hRBC}$ in PBS, and $100 \mathrm{~mL}$ of peptide solution was added to each well. Plates were incubated for $1 \mathrm{~h}$ at $37^{\circ} \mathrm{C}$, then centrifuged at $1000 \times \mathrm{g}$ for $5 \mathrm{~min}$. Samples $(100 \mathrm{~mL})$ of supernatant were transferred to 96-well plates and hemoglobin release was monitored by measuring absorbance at $414 \mathrm{~nm}$. Zero hemolysis was determined in PBS (A $\mathrm{APS}_{\mathrm{PBS}}$ ) and 100\% hemolysis was determined in $0.1 \%(\mathrm{v} / \mathrm{v})$ Triton X-100 $\left(\mathrm{A}_{\text {triton}}\right)$. The hemolysis percentage was calculated as: $\%$ hemolysis $=100 \times\left[\left(\mathrm{A}_{\text {sample }}-\mathrm{A}_{\mathrm{PBS}}\right) /\left(\mathrm{A}_{\text {triton }}-\mathrm{A}_{\mathrm{PBS}}\right)\right]$

Dye Leakage. Calcein-entrapped large unilammelar vesicles (LUVs) composed of EYPE/EYPG (7:3, w/w) were prepared by vortexing the dried lipid in a dye buffer solution (70 mM calcein, $10 \mathrm{mM}$ Tris, $150 \mathrm{mM} \mathrm{NaCl}, 0.1 \mathrm{mM}$ EDTA, pH 7.4). The suspension was subjected to 10 freezethaw cycles in liquid nitrogen and extruded 21 times through polycarbonate filters ( 2 stacked $100-\mathrm{nm}$ pore size filters) with a LiposoFast extruder (Avestin, Inc. Canada). Untrapped calcein was removed by gel filtration on a Sephadex G-50 column. The concentration of calcein-entrapped LUVs was determined in triplicate by phosphorus analysis. Calcein leakage from LUVs was monitored by measuring the fluorescence intensity at an excitation wavelength of $490 \mathrm{~nm}$ and emission wavelength at $520 \mathrm{~nm}$ on a model RF-5301PC spectrophotometer. Complete dye release was obtained using $0.1 \%$ Triton X-100.

Transmission Electron Microscopy (TEM). Following the addition of AMPs, the samples were incubated for $1 \mathrm{~h}$ at $37^{\circ} \mathrm{C}$. Bacterial cell pellet after centrifugation was resuspended in $20 \mu \mathrm{L}$ PBS for TEM specimen preparation. 5 $\mu \mathrm{L}$ of sample solution was loaded onto a carbon film-coated TEM grid that was rendered hydrophilic by glow discharge. After 90 seconds, excess sample solution was washed off with distilled water. $5 \mu \mathrm{L}$ of $1 \%$ uranyl acetate was loaded onto the grid for negative staining for 1 minute, and excess stain solution was blotted using a piece of filter paper. Samples were imaged using a JEM-1400Plus electron microscope (JEOL) equipped with lanthanum hexaboride $\left(\mathrm{Lab}_{6}\right)$ gun, operating at $120 \mathrm{kV}$. Images were recorded using 8 mega pixel, bottom-mount charge-coupled device (CCD) camera (EM-14650DR13, JEOL).

Circular Dichroism (CD) Spectroscopy. The circular dichroism (CD) spectra of the peptides were recorded at 25 ${ }^{\circ} \mathrm{C}$ using a Jasco J-715 CD spectrophotometer (Tokyo, Japan). The samples were scanned at room temperature in a capped quartz cuvette (1-mm path length) cells in the wavelength range of 190-250 nm. The spectra were recorded at a peptide concentration of $100 \mu \mathrm{g} / \mathrm{mL}$ in $10 \mathrm{mM}$ sodium phosphate buffer (pH 7.2) and $30 \mathrm{mM}$ sodium dodecyl sulfate (SDS) micelles. The mean residue ellipticity, $[\theta]$, was given in $\operatorname{deg} \cdot \mathrm{cm}^{2} \cdot \mathrm{dmol}^{-1}:[\theta]=[\theta]_{\text {obs }}(\mathrm{MRW} / 10 \times 1 \times \mathrm{c})$, where: $[\theta]_{\mathrm{obs}}$ is the ellipticity measured in millidegrees, MRW is the mean residue molecular weight of the peptide, $\mathrm{c}$ is the concentration of the sample in $\mathrm{mg} / \mathrm{mL}$, and 1 is the optical path length of the cell in $\mathrm{cm}$. The spectra were expressed as molar ellipticity $[\theta] v s$. wavelength.

\section{Results}

Preparation of Pac-525 and Its Analogues. Pac-525 (Ac-KWRRWVRWI-NH $_{2}$ ) was used as model compound because Pac-525 has each 3 Trp and 3 Arg residues among the 9 sequence positions which are considered as Trp- and Arg-rich AMP, moreover they are highly active even at short peptide length. In order to study the properties of Trp residues in Pac 525, were examined with derivatives obtained by alanine (Ala) substitution of Trp moiety namely, (i). the mono-substitution of alanine at 2, 5 and 8 position (positions 2, 5 and 8) resulted in $\mathrm{P} 2, \mathrm{P} 3$ and $\mathrm{P} 4$, respectively, (ii). Alanine analogs were derived by the di-substitution at various positions $(2 \& 5),(5 \& 8)$ and $(2 \& 8)$ led to the formation of P5, P6 and P7 respectively, (iii). Tri-substitution of alanine at $(2,5 \& 8)$ derived the P8. Subsequently, Pac-525 analogues having the His(chx) were synthesized and their biological activities were examined. The monosubstitution of His(chx) with Trp residue at P5, P6 and P7 resulted in the formation of $\mathrm{P} 9, \mathrm{P} 10$ and $\mathrm{P} 11$ respectively. Finally, we synthesized Pac-525 shorten by deleting Cterminal 5 residues known as P15 in which Trp were substituted by His(chx) derived P16 as to compare the biological activities (Table 1).

Antibacterial and Hemolytic Activities. All of the peptides were assayed for their antibacterial activities towards two Gram-negative bacteria (E. coli, and P. aeruginosa) and two Gram-positive bacteria (S. epidermidis, and S. sureus). The cytotoxicity of the peptides was evaluated by testing hemolysis with human red blood cells (hRBCs). For the purpose of comparison, the previously reported active AMPs, Pac-525 and melittin, were served as a positive control for antibacterial and hemolytic activities. The MIC values of Pac-525 and the analogues are shown in Table 2. In the cases of mono-substituted Trp residues, P4 showed $\sim 2-4$ fold increase in antibacterial activity against all the bacterial strains except $S$. aureus remained as same and in terms of P2 and $\mathrm{P} 3$ retained their activity in all strains excluding $P$. aeruginosa, displayed 2-fold increase in activity (supplementary table S1). However, di-substituted Trp analogues $\left[\mathrm{Ala}^{5,8}\right] \mathrm{Pac}-525$ (P6) lost their activity by 2 -fold against all the bacterial strains, similarly $\left[\mathrm{Ala}^{2,5}\right] \mathrm{Pac}-525$ (P5) showed decrease in activity by 2 -fold against all bacterial strains except $S$. epidermidis, and $\left[\mathrm{Ala}^{2,8}\right] \mathrm{Pac}-525$ (P7) lost their activity by 2 -fold against $E$. coli and $S$. aureus, meanwhile retained their activity against $P$. aeruginosa and $S$. epidermidis. In contrast, tri-substituted Trp analogue $\left[\mathrm{Ala}^{2,5,8}\right] \mathrm{Pac}-$ 525 (P8) lost their activity entirely towards both Gramnegative and Gram-positive bacterial strains. Results evolved from the replacement of Trp residues suggested to the conclusion that Trp residues play dynamic role in the determination of antibacterial activity in Pac-525. 
Table 1. Amino acid sequences of P525 and its analogs

\begin{tabular}{|c|c|c|c|}
\hline \multirow{2}{*}{ Peptides } & \multirow{2}{*}{ Amino acid sequences } & \multicolumn{2}{|c|}{ Molecular mass (MS) } \\
\hline & & Calculated & Observed $^{a}$ \\
\hline \multicolumn{2}{|c|}{ P525 (P1) Ac-KWRRWVRWI-NH ${ }_{2}$} & 1426 & 1427 \\
\hline $\mathrm{P} 2$ & Ac-KARRWVRWI-NH ${ }_{2}$ & 1311 & 1312 \\
\hline P3 & Ac-KWRRAVRWI-NH ${ }_{2}$ & 1311 & 1312 \\
\hline P4 & Ac-KWRRWVRAI-NH ${ }_{2}$ & 1311 & 1312 \\
\hline P5 & Ac-KARRAVRWI-NH 2 & 1196 & 1198 \\
\hline P6 & Ac-KWRRAVRAI-NH ${ }_{2}$ & 1196 & 1197 \\
\hline P7 & Ac-KARRWVRAI-NH ${ }_{2}$ & 1196 & 1197 \\
\hline P8 & Ac-KARRAVRAI-NH ${ }_{2}$ & 1081 & 1082 \\
\hline P9 & Ac-KARRAVRHis(chx)*I-NH${ }_{2}$ & 1396 & 1396 \\
\hline $\mathrm{P} 10$ & Ac-KHis(chx)RRAVRAI-NH 2 & 1396 & 1396 \\
\hline P11 & Ac-KARRHis(chx)VRAI-NH ${ }_{2}$ & 1396 & 1396 \\
\hline P15 & Ac-KWRR-NH ${ }_{2}$ & 685 & 687 \\
\hline P16 & Ac-KHis(chx)RR-NH ${ }_{2}$ & 886 & 887 \\
\hline
\end{tabular}

${ }^{a}$ Molecular mass were determined using matrix-assisted laser desorption/ ionization, time-of-flight mass spectrometry. ${ }^{*} \mathbf{H i s}(\mathbf{c h x})$

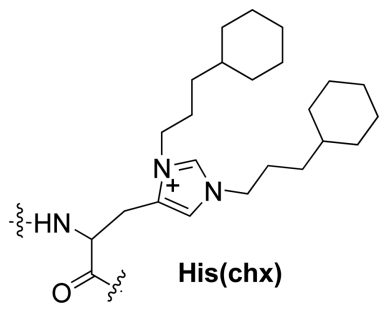

In order to investigate the effect of His(chx), P9, P10 and P11 were analyzed. In the case of $\mathrm{P} 9\left(\left[\mathrm{His}(\mathrm{chx})^{8}\right]\right)$, when compared to Pac525(P1), there was a increase in activity by 2 -fold against all strains tested meanwhile P10([His $\left.\left.(\mathrm{chx})^{2}\right]\right)$ displayed 2-8 fold increase in activity. Similarly P11([His$\left.\left.(\operatorname{chx})^{5}\right]\right)$ also showed potential increase in activity by 8 fold against Gram-positive bacteria and 4 fold against Gramnegative bacteria while hemolytic activity did not show at a concentration as high as $256 \mu \mathrm{g} / \mathrm{mL}$. This suggested that the positional shift of His(chx) was not in favour of determining the selectivity rather it participates towards increasing the antibacterial activity. Finally, the short peptide P15 antibacterial activity assay result disclosed that there was entire loose of activity, can be attributed to minimal length of peptide chain. Surprisingly, His(chx) based P16 exhibited the most potential antibacterial activity towards both Gramnegative and Gram-positive bacteria. It is much potent than melittin despite P16 consists of only 4 amino acid residues meanwhile hemolytic activity was absent even at a concentration of $256 \mu \mathrm{g} / \mathrm{mL}$. Obviously these results suggested to conclude that, the substitution Trp with His(chx) may endow balanced hydrophobicity and charge, which might be took part in the enhancement of antibacterial activity. Considering all these facts, we can confirm that the His(chx) could be of the appropriate candidate for replacing the Trp which can be applied to design the potential AMP. It is pertinent to note that none of candidate either Pac-525 or its analogues disclosed no hemolytic activity even at the highest concentration $(256 \mu \mathrm{g} / \mathrm{mL})$.

Antibacterial Activity Against MRSA. For past decades, the annual frequency of deaths from MRSA has been rapidly increasing. ${ }^{16}$ In addition to the broad spectrum of antibacterial activity described above, next we screened the anti-MRSA activity against MRSA strains for His(chx) based P10, P11 and P16 (Table 3). The assay results revealed some fascinating aspects that P10 and P11 displayed good anti-MRSA activity employed for all MRSA strains. Furthermore, the shortest peptide P16 revealed good activity as same as P10

Table 3. Antimicrobial activity of P525 analogs against methicillin-resistant Staphylococcus aureus (MRSA) strains

\begin{tabular}{cccc}
\hline \multirow{2}{*}{ Peptides } & \multicolumn{3}{c}{ Minimal Inhibitory Concentration (MIC) ${ }^{a}$} \\
\cline { 2 - 4 } & $\begin{array}{c}\text { MRSA 1 } \\
\text { (CCARM 3089) }\end{array}$ & $\begin{array}{c}\text { MRSA 2 } \\
\text { (CCARM 3090) }\end{array}$ & $\begin{array}{c}\text { MRSA 3 } \\
\text { (CCARM 3095) }\end{array}$ \\
\hline P10 & 8 & 8 & 8 \\
P11 & 8 & 8 & 8 \\
P16 & 4 & 8 & 8 \\
\hline
\end{tabular}

${ }^{a}$ Minimal inhibitory concentration of peptides in $\mu \mathrm{g} / \mathrm{mL}$

Table 2. Antimicrobial and hemolytic activities of P-525 and its analogs

\begin{tabular}{|c|c|c|c|c|c|}
\hline \multirow[b]{2}{*}{ Peptides } & \multicolumn{4}{|c|}{ Minimal Inhibitory Concentration (MIC) $(\mu \mathrm{g} / \mathrm{mL})$} & \multirow{2}{*}{$\begin{array}{l}\text { \% Hemolysis } \\
(256 \mu \mathrm{g} / \mathrm{mL})\end{array}$} \\
\hline & $\begin{array}{c}\text { E. coli } \\
\text { [KCTC 1682] }\end{array}$ & $\begin{array}{l}\text { P. aeruginosa } \\
\text { [KCTC 1637] }\end{array}$ & $\begin{array}{l}\text { S. epidermidis } \\
\text { [KCTC 1917] }\end{array}$ & $\begin{array}{c}\text { S. aureus } \\
\text { [KCTC 1621] }\end{array}$ & \\
\hline P525 (P1) & 32 & 32 & 32 & 32 & 0 \\
\hline P5 & 64 & 64 & 32 & $>64$ & 0 \\
\hline P6 & 64 & 64 & 64 & $>64$ & 0 \\
\hline P7 & 64 & 32 & 32 & $>64$ & 0 \\
\hline P8 & $>64$ & $>64$ & $>64$ & $>64$ & 0 \\
\hline P9 & 16 & 16 & 16 & 16 & 0 \\
\hline P10 & 8 & 16 & 8 & 4 & 0 \\
\hline P11 & 8 & 8 & 4 & 4 & 0 \\
\hline P15 & $>64$ & $>64$ & $>64$ & $>64$ & 0 \\
\hline P16 & 4 & 8 & 4 & 4 & 0 \\
\hline Melittin & 16 & 16 & 4 & 4 & $100^{a}$ \\
\hline
\end{tabular}

${ }^{a}$ Melittin induced $100 \%$ hemolysis at $32 \mu \mathrm{g} / \mathrm{mL}$ 

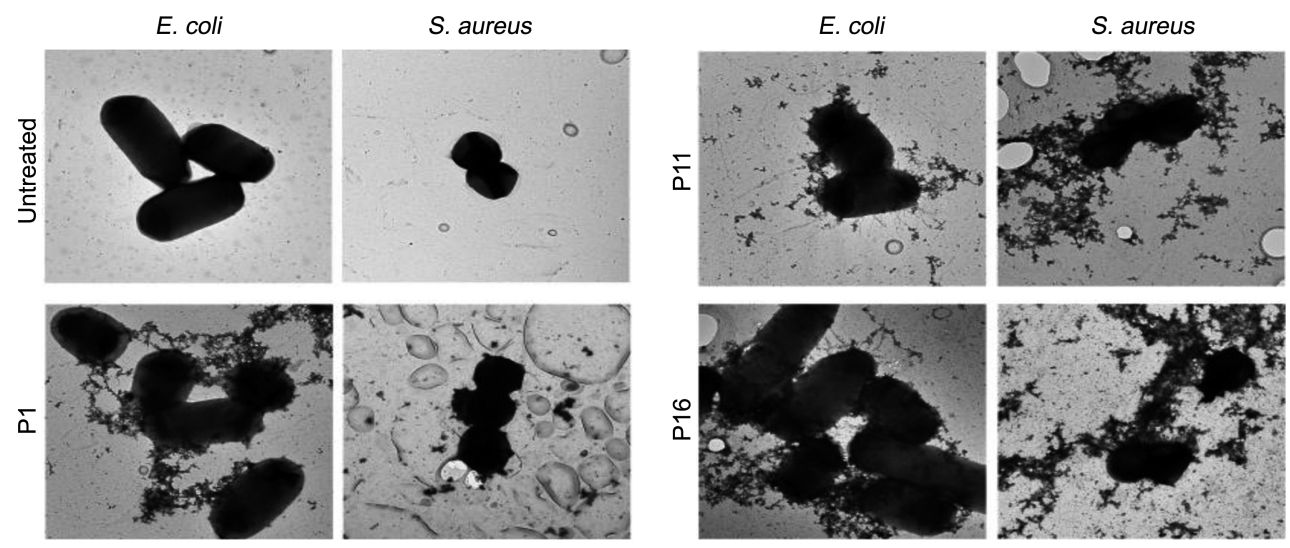

Figure 1. Electron micrographs of negatively stained E. coli and S. aureus untreated or treated with P1, P11 and P16. All images are scaled, and the scale bar represents $1 \mu \mathrm{m}$.

and P11 against MRSA 2 and MRSA 3 strains, surprisingly, while it displayed 2 fold increase in potential activity against MRSA 1.

Transmission Electron Microscopy (TEM). As a brief study of the mechanism of action, morphological changes in bacterial strains, E. coli and $S$. aureus, upon the addition of designed Pac-525 based analogues were examined using transmission electron microscopy (TEM). In addition, p525 (Pac-525) was also tested for bacterial lysis as a positive control. In comparison to untreated control, obvious disruption of cellular structure was observed when P11 and shortest peptide P16 were added (Figure 1). It is pertinent to note that clear rupturing of bacterial cells and release of internal materials were clearly visible, while the structures of untreated cells were preserved without any indication of cell damage.

Dye Leakage. To determine whether the antibacterial activities of the peptides depend on their capacity to permeate bacterial membranes, we measured their abilities to induce the fluorescent dye calcein leakage from negatively charged phosphatidylethanolamine (PE)/phosphatidylglycerol (PG) (7:3, w/w) large unilamellar vesicles (LUVs), which mimics bacterial membranes. As compared to melittin, P11 and P16

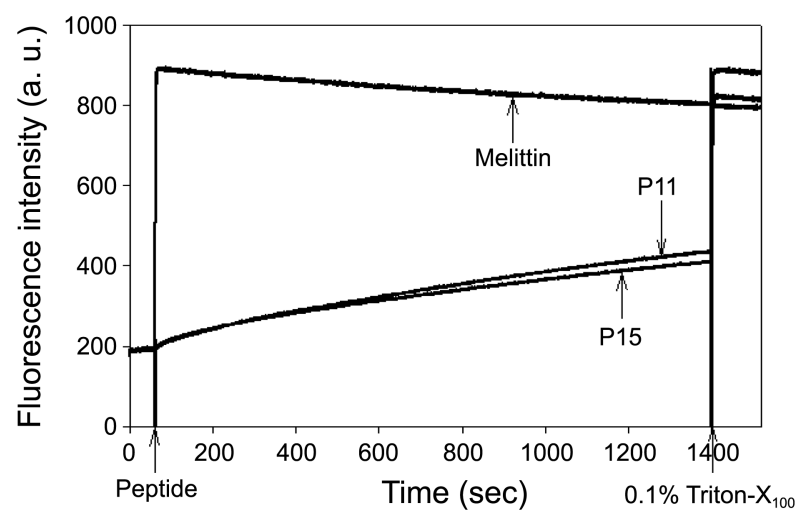

Figure 2. Peptide-induced dye leakage from calcein-entrapped negatively charged phosphatidylethanolamine (PE)/phosphatidylglycerol (PG) (7:3, w/w) large unilamellar vesicles (LUVs). Peptide concentration: $16 \mu \mathrm{g} / \mathrm{mL}$. induced relatively low (less than 40\%) calcein leakage at 16 $\mathrm{mg} / \mathrm{mL}$ (Figure 2). This relatively lower calcein leakage from LUVs suggests that P11 and P16 may act by forming pores, instead of disrupting the bacterial membrane.

Circular Dichroism (CD). We compared the secondary structure of the Pac-525 and its analogues using CD spectro-
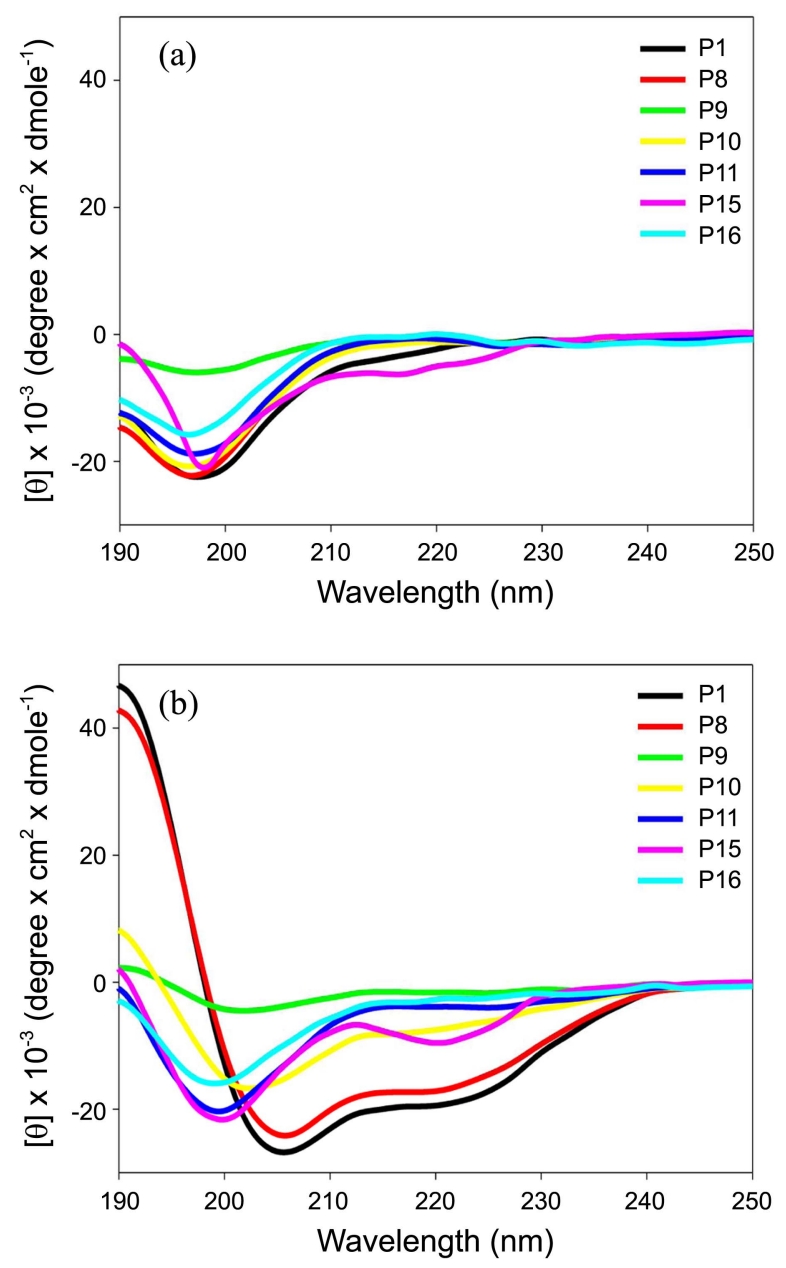

Figure 3. CD spectra of the peptides in sodium phosphate buffer (pH 7.2) (a) and $30 \mathrm{mM}$ sodium dodecyl sulfate (SDS) micelles (b). 
scopy (Figure 3). CD spectra were taken in sodium phos-

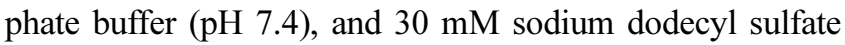
(SDS). In the sodium phosphate buffer, Pac-525 and all its analogues were characterized by a broad negative band of around $200 \mathrm{~nm}$, characteristic of an unordered structure. In SDS environment, Pac-525 and P8 showed the dramatic structure change resulting in an ordered a-helix structure. This was supported by the fact that increasingly negative bands at 208 and $220 \mathrm{~nm}$ were observed in the presence of SDS. In contrast, His(chx)-substituted P10, P11 and P12 displayed the random coil patterns. Consequently we concluded that insertion of His(chx) in P8 changed the conformation from a-helix into random coil. In addition, P15 and P16 showed the random coil because of their short peptide length in nature.

\section{Discussion}

Trp residue are considered as one of the important constituents in AMP as it presence secured good activities in many cases. However, it is preferable to be changed because of their sensitivity towards light. In this context the present investigation emerged as to explore unnatural amino acid to replace the Trp residue. We have synthesized the Pac-525 and its analogues arose from the replacement of Trp with His(chx). In addition, to address the influence of Trp residue on Pac-525 antibacterial properties, analogues were derived from the substitution of alanine (Ala) with Trp in Pac-525. According to MIC results, a Trp analogues substituted at 2 and 5 position (positions 2 and 5) viz $\mathrm{P} 2$ and $\mathrm{P} 3$ with Ala, showed minimum or no effect on their antibacterial activity and hemolysis while P4 analogue was disclosed considerable antibacterial activity. Furthermore, the antibacterial activity of di-substituted analogues $\left[\mathrm{Ala}^{2,5}\right] \mathrm{Pac}-525$ (P5) and $\left[\mathrm{Ala}^{5,8}\right] \mathrm{Pac}-525(\mathrm{P} 6)$ decreased dramatically, proving the presence of couple of Trp residues are crucial for the biological activity except for $\left[\mathrm{Ala}^{2,8}\right] \mathrm{Pac}-525$ (P7). But in the case of the analogue in which all Trp residues were substituted with Ala, $\left[\mathrm{Ala}^{2,5,8}\right] \mathrm{Pac}-525$ (P8), lost its activity completely. Next, we replaced the Trp residue of Pac 525 with His(chx) and tested their antibacterial activities. MIC results showed that the introduction of the unnatural amino acid His(chx) as replacement of Trp of Pac-525 gave a dramatic increase in the antibacterial activity without hemolytic activity. In contrast to P6 and P7 having one Trp in the Pac-525 sequence, P10 and P11 having one His(chx) instead of Trp showed the more higher antibacterial activity toward both Gram-positive and Gram-negative bacteria than Pac525. Surprisingly, P16 composing of one His(chx) and three Lys amino acids showed the potent antibacterial activity while P15 composing one Trp and three Lys amino acids failed to show any promising activity. It is evident from the antibacterial activities of the all compounds His(chx) can be a good candidate for the Trp replacement as to design potential antimicrobial agents.

Finally, P10, P11 and P16 with potent antibacterial activity were subjected to anti-MRSA assay to investigate the potential for their therapeutic application. Remarkably, the result of MRSA assay suggested that the selected Pac-525 analogues (P10, P11 and P16) overcame the development of MRSA resistance (Table 3). Moreover, according to the secondary structure of the Pac-525 and its analogues using CD spectroscopy, the data clearly showed that His derivative having cyclohexyl pendant alkyl tail participates in destroying their a-helix structure resulting in random coil patterns. In addition, based on the mechanistic studies (Figures 1 and 2), we propose here that P11 and P16 containing His(chx) kill bacteria via forming pore/ion channels on bacterial cell membranes.

\section{Conclusion}

Through these study, we can conclude that His(chx) has several merits to design therapeutic antimicrobial agents compare to Trp in terms of i) increasing antibacterial activity without hemolytic activity, ii) successful in designing the short peptide (only four residues), iii) having anti-MRSA activity, vi) overcoming the light sensitivity.

Acknowledgments. This work was supported in part by Korea Basic Science Institute's research grant T34418 (J.K.B), the Next-Generation BioGreen 21 Program (\#PJ009594, N.H.K), Rural Development Administration, Republic of Korea and Korea Research Foundation funded by the Korean Government (KRF-2011-0009039 to S.Y.S.).

\section{References}

1. O'Connell, K. M.; Hodggkinso, J. T.; Sore, H. F.; Welch, M.; Salmon, G. P.; Spring, D. R. Angew. Chem. Int. Ed. 2013, 52, 10706.

2. Bush, K.; Courvalin, P.; Dantas, G.; Davies, J.; Eisentein, B.; Huovine, P.; Jacoby, G. A.; Kishony, R.; Kreiswirth, B. N.; Kutter, E.; Lehner, S. A. Nat. Rev. Microbiol. 2011, 9, 894.

3. Ganz, T. Nat. Rev. Immunol. 2003, 3, 710.

4. Nessar, R.; Cambau, E.; Reyrat, JM.; Murray, A.; Gicqel, B. J. Antimicrb. Chemother. 2012, 67, 810.

5. Reddic, L. E.; Alto, N. M. Mol. Cell 2014, 54, 321.

6. Zasloff, M. Nature 2002, 415, 389.

7. Tossi, A.; Sandri, L. Curr. Pharm. Des. 2002, 8, 743.

8. (a) Kuriakose, J.; Hernadez-Gordillo, V.; Nepal, M.; Brezden, A.; Pozzi, V.; Seleem, M. N.; Chmielewski, J. Angew. Chem. Int. Ed. 2013, 52, 9664. (b) Lee, E.; Kim, J.; Shin, S.; Jeong, K.; Shin, A.; Lee, J.; Lee, D.; Hwang, J.; Kim, Y. Biocimica et Biophysica Acta 2013, 1828, 271. (c) Chan, D. I.; Prenner, E. J.; Vogel, H. J. Biocimica et Biophysica Acta 2006, 1758, 1184.

9. Ando, S.; Mitsuyassu, K.; Soeda, Y.; Hidaka, M.; Ito, Y.; Matsubara, K.; Shindo, M.; Uchida, Y.; Aoyagi, H. J. Pept. Sci. 2010, 16, 171,

10. (a) FimLand, G.; Eijsink, V. G.; Nissen-Meyer, J. Biochemistry 2002, 41, 9508. (b). Schibli, D. J.; Epand, R. F.; Vogel, H. J.; Epand, R. M. Biochem Cell Biol. 2002, 80, 667.

11. Sharma, R. K.; Reddy, R. P.; Tegge, W.; Jain, R. J. Med. Chem. 2009, 52, 7421 .

12. Haug, B. E.; Skar, M. L.; Svendsen, J. S. J. Pept. Sci. 2001, 7, 425.

13. Subbalakshmi, C.; Krishnakumari, V.; Nagaraj, R.; Sitaram, N. FEBS Lett. 1996, 395, 48.

14. (a) Ahn, M.; Murugan, R. N.; Jacob, B.; Hyun, J. K.; Cheong, C.; Hwang, E.; Park, H. N.; Seo, J. H.; Srinivasrao, G.; Lee, K. S.; Shin, S. Y.; Bang, J. Eur. J. Med. Chem. 2013, 68, 10. (b) 
Murugan, R. N.; Jacob, B.; Kim, E. H.; Ahn, M.; Seo, J. H.; Cheong, C.; Hyun, J. K.; Lee, K. S.; Shin, S. Y.; Bang, J. Bioorg. Med. Chem. Lett. 2013, 23, 4633. (c) Murugan, R. N.; Jacob, B.; Ahn, M.; Hwang, E.; Sohn, H.; Park, H. N.; Lee, E.; Seo, J. H.; Cheong, C.; Nam, K. Y.; Hyun, J. K.; Jeong, K. W.; Kim, Y.; Shin, S. Y.; Bang, J. PLoS One 2013, 8, e80025.

15. (a) Li, H.; Cheng, J. W.; Yu, H. Y.; Xin, Y.; Tang, L.; Ma, Y. J.
Microbiol. Biotechnol. 2013, 23, 1070. (b) Qi, X.; Zhou, C.; Li, P.; Xu, W.; Cao, Y.; Ling, H.; Ning Chen, W.; Ming Li, C.; Xu, R.; Lamrani, M.; Mu, Y.; Leong, S. S.; Wook Chang, M.; Chan-Park, M. B. Biochem. Biophys. Res. Commun. 2010, 30, 594.

16. Menousek, J.; Mishra, B.; Hanke, M. L.; Heim, C. E.; Kielian, T. Int. J. Antimicrob. Agents. 2012, 39, 402. 